\title{
SIXTH SENSE TECHNOLOGY FOR HOME AUTOMATION
}

\author{
Zeba Raut ${ }^{1}$ \\ ${ }^{I}$ Student, Electronics Dept., Pillai College of Engineering, Maharashtra, India
}

\begin{abstract}
It is a beginning of a distinctive point of time of technology where engineering will reach new development. Similar to what we witness in the movies where computer screens are displayed on walls, where instructions are given by gestures, the digital information which directly interact with us to do daily activities and so on, these can be made to implement in reality very soon by using sixth sense technology. Earlier this type of implementation of the technology in the movies was considered as imaginative or supernatural. Now, there is no need for these science fiction movie makers to think ahead of time because the technology shown in such movies can be even in the household. Sixth sense is an interface that inculcate the digital information with the physical world and helps us to communicate with this information. It is based on concepts that denotes reality as well as execute the profundity of it. Since, the digital world has become equally important to us than the physical world it has become necessary for a person to learn it so as to communicate. This type of technology has brought together the physical world objects with digital world. Sixth sense is more about encompassing information more than our own five senses. And today digital world too has become an important part of our life. This digital world is equally important to all of us. Sixth sense technology helps in analyzing this digital world in an easy and more straightforward approach.
\end{abstract}

Keywords: Sixth Sense, Digital Information, Physical World.

\section{INTRODUCTION}

We have developed gradually over years to sense the world. When we witness something we use our five senses eye, ear, nose, tongue, mind and body to interpret information which helps us to make the correct decision. Sixth sense receives the information not gained by any of the five senses. It is explained in terms of scientific terms known as Extra Sensory Perception or ESP. This project deals with the latest technology called the sixth sense. The digital information is augmented with the physical world around us with the help of a portable interface. Nowadays, there is no connection between our digital devices and their communication with the physical world. Sixth sense bridges this gap, it brings the impalpable physical information into palpable physical world and allows users to interact with this information via images. It frees the information that is restricted by combining it with the reality. Thus, it allows users to connect with the real world. It is a technology with which the device could be trained recognize and interpret real world objects and react as desired. In this project, the users show images drawn on paper to the web camera of the laptop. It creates a machine vision and image processing application using LabView VI. Machine vision permits the computer to capture images.

These images are used to turn on or turn off the electronic devices. This can be done in establishing client-server network for data transmission. The Raspberry PI acts as a server which receives information based as per the images from the client through TCP/IP connection. It is connected to the hardware devices which receives information sent by it and operates accordingly.

\subsection{Literature Survey}

In [1], in this paper there is a brief summary in the field of technology based on sixth sense. Sixth sense is a portable interface which combines the physical world around us with digital data and provides us a chance to use the signals to interact with the digital information. The goal is to review software, components used in this type of technology and its application.

In [2], In image acquiring by using sixth sense technology the conversion of real world into digital world is possible. It allows computing and development and external environment to provide information controls. A large amount of equipments are used these days for capturing pictures like camera with memory cards, cell telephones and so on.

In [3], small scale computing devices helps us to be constantly in contact with the digital environment. Sixth sense technology is a transpiring trend, In order to overcome the physical reliance on traditional paper, hardware devices, variety of applications are developed using this technology. Thus, it emancipates the restrained information from such platforms. This paper provides the idea of designing input keyboard and paint using hand gestures or symbols. By, using this user can control any type of application.

\subsection{Problem Definition}

Why choose sixth sense?

Sixth sense is defined in scientific terms as Extra sensory perception or ESP. It includes receiving information not acquired by any of the five senses. This can be made possible by allowing users to perform decision making with that information which is available beyond which they have 
access to the five senses. Thus it helps in efficiently giving users sixth sense.After acquisition of information from the available senses as its inputs it starts the operation. But the information that is collected isn't enough to make the correct decisions. However, the information which could make use of to make correct decision is abundantly available on the internet. By connection of the devices like for example mobiles, laptops or computers we can collect the required information but this information is confined to the screen and we cannot directly communicate the impalpable digital world with the palpable physical world. This type of technology gives users us the freedom to communicate with the digital world with hand gestures, images or other symbols. The sixth sense technology can also be used on a large scale in the field of artificial intelligence. This technology can help to synthesize bots that can easily interact with human beings.

Sixth sense technology is an interpretation of reality. Like senses enable us to recognise information about the world around us in variety of way, it also aims at sensing the information. Nowadays, there is not just the physical world we interact with but also the digital world which has equally become an important part our life with the use of internet, the digital world can be enlarged many times compared to the physical world. There is no way we can interact with the digital world so we created them like smart phones, computers, laptops, tablet and other electronic equipments. These enable us to communicate with the digital world.

\section{METHODOLOGY}

- Simulation Tool : LabView, Python

- Hardware components : ARM11 Raspberry PI board, Web camera(laptop), Regulated power supply, Relay driver circuit, Relay driver IC.

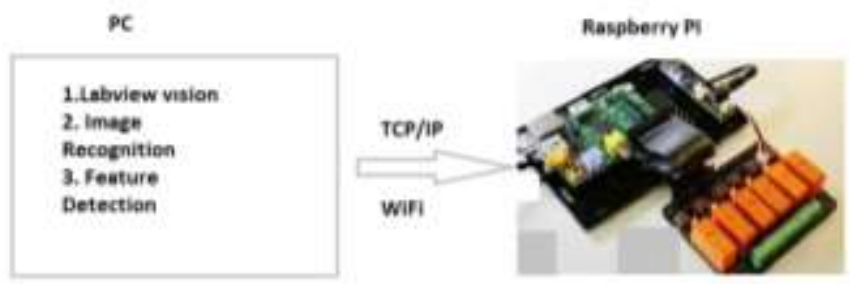

Fig -1: Name of the figure

The project deals with the client server network where laptop acts as a client and Raspberry Pi acts as a server and they communicate with each other through TCP/IP. The web camera of the laptop captures the images drawn on paper and sends the information based on these images to the Raspberry Pi circuit on its GPIO lines which acts as an output. It is given to the relay driver circuit where hardware devices are connected across the relays. These electronic devices are turned on or off based on the information to the server.

Programming languages: LabView is used to analyse, detect, and display images. It is a system design environment for visual programming languages from national instruments. It uses image processing functions by developing machine vision application.Python is a developing tool, it is a combination of $\mathrm{C}$ and $\mathrm{C}++$. It is widely used interpreted language where the programmer does not require additional programming files for implementation.

Memory Management: Python is a dynamic programming language with automatic memory management. It has a large and inclusive library. LabView has a graphical source coding with large library. It inculcates parallel programming.

Circuit diagram and description:The major building blocks of this project are:

- ARM11 Raspberry Pi board.

- Web Camera(Laptop)

- Regulated power supply.

- Electromagnetic relays to control devices.

- Relay Driver IC.

Camera:Images can be captured and recognized by using a webcam of PC or a laptop which detects the images using image processing technique. The information based on the images can be sent to the smart phone. The camera, operates and performs as a digital eye, as it visualizes the same thing what users visualize. The camera perceives objects around us instantly, this is done with the help of a micro projector overlaying the information on any surface, which includes your hand or the object itself.

\section{ARM11 Raspberry PI Board}

-The Raspberry Pi foundation in the UK has invented and developed Raspberry Pi hardware in order to promote the knowledge and teaching of the basic computer science. Raspberry $\mathrm{Pi}$ is defined as a series of credit card sized system on chip based computers.

The Raspberry Pi which was original is based on the BCM2835 Broadcom system on a chip, it includes an Video Core IV GPU, 512Mb of RAM, ARM1176JZF-700 MHz processor.

The Raspberry Pi Foundation provides Arch Linux and Debian ARM distributions for downloads. Tools that are available for the main programming language such as Python, with the support for C, C++, Java, Perl and Ruby.

Hardware Functional Block:

The Raspberry Pi hardware has enhanced through several versions that features:

- $700 \mathrm{MHz}$ ARM1176JZFS processor

- Video Core IV GPU

- Level 1 cache of $16 \mathrm{~KB}$ and a

- Level 2 cache of $128 \mathrm{~KB}(\mathrm{GPU})$

- Variations in hardware performance, memory capacity, and peripheral device support.

- Broadcom BCM2835 system on a chip (SoC) 


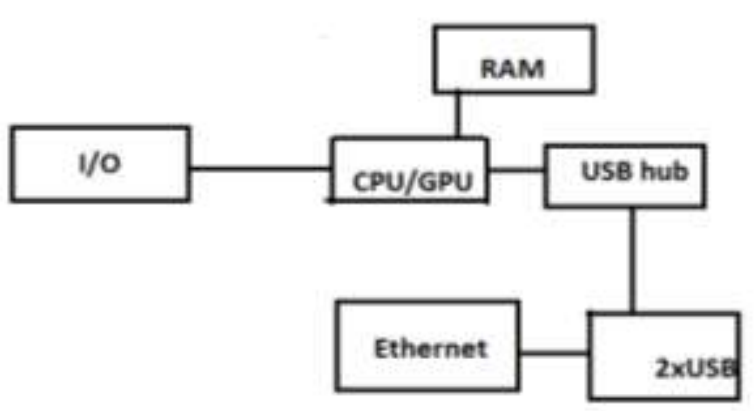

Fig-2: Hardware Functional Block of Raspberry Pi

\section{Relay Driver IC Circuit}

Relays enables a low power circuit to control signals or to shift high current $\mathrm{ON}$ and OFF which should be electrically secluded from the controlling circuit. Relays are elements that are useful when there is a need to control large amount of current or voltage.

Let us observe the fabrication of relay driver circuit for relays. The operation of relays is carried out from DC power. To rate a relay and a zener diode, DC voltage is required in a particular quantity. DC voltage is used to in order for the operation of relay. for relay to operate voltage is required and to close or open its switch in a circuit. Relays comes with a voltage rating. Relays datasheet is used to rate its coil voltage. The voltage should first appear across the coil of relay for it to function. Thus, for example if a relay has a rated voltage of $10 \mathrm{VDC}$, it should get 10 volts of DC voltage for it to properly work. Voltage spikes appear across the coil so to eliminate the spikes we make use of a diode which carries a reverse current in an appropriate quantity. The Relay coil operates as an Inductor.

The inductors are electronic substance which resists strong changes in current and it possesses inductance which are wires intertwined to form coils that are wrapped around a core which is conductive in nature. A transistor should be used to drive a relay so we make use of a transistor that consumes less power to drive a relay. The transistor acts as an amplifier so in order to make current flow from emitter to collector of the transistor the base lead has to receive sufficient current.

For amplification or boosting the signals a driver circuit is used to regulate power switches in various electronic equipments. Driver circuits operates to perform various functions which includes seclusion of the power circuit and control circuit, precaution from failure, in order to detect malfunctions, for sensor signals to be analysed and for creating low voltage circuitry. Voltage spikes need to be controlled as it can damage all the circuit components and it can also damage switch contacts of relays Thus prevention from such voltage spikes is essential in order to protect the circuit from getting damaged.

A diode is placed in a reverse biased condition which is kept parallel to the relay so as to prevent the voltage spikes. It functions as a transient (spike) suppressor in order to eliminate the voltage spikes. They achieve this by conducting voltage across it first before the voltage appears across the coil terminals of the relays. In such a way it is able to suppress the voltage spikes. A certain threshold of an appropriate quantity is required for the diode to conduct a reverse bias current. So, whenever the voltage reaches the threshold value the diode conducts a reverse current. The diode functions to shunt The excess power is shunted to the ground with the help of a diode, and when the voltage reaches breakdown voltage they go into the state of conduction.

Advantages of a Relay driver IC:

This consists of widely available IC driver which is ULN2003A

Low voltage circuitry can be easily interfaced.

The components used are less in number.

NPN drive transistors are less expensive.

Load on voltage regulator is reduced by relays.

NPN drive transistors that are commonly available are used. It is possible to reset a relay fast and it acts spontaneously and quickly.

Relays are easier to construct and are reliable.

It makes use of standard industry technique.

Easier for interfacing relays.

It is highly economic for its purpose.

The components which are required in this circuitry are:

DC power supply or DC source voltage.

DC relay.

Zener Diode.

The relay is kept parallel to the zener diode.

A 9 volts rated relay is used in this circuit. The resistor is fed to a $9 \mathrm{~V}$ DC source voltage. In order to suppress the transients that is the voltage spikes a zener diode is kept in parallel with the relay. It carries out its operation by closing and opening the relay and this causes all the excess power to be shunted to the ground thus, it protects the circuit from getting damaged. The relay will be closed which drives the output connected to the load by taking the required power.

\section{Relay Driver IC ULN2003A}

The relay driver ULN2003A IC is a current darlington array IC and has a high voltage, which is comprised of common emitters with 7-open collector darlington pairs. Two bipolar transistors arrangement is required to form a darlington pair. It interfaces to varied type of logic families and this IC belongs to the family of ULN200x. 5V TTL and CMOS logic devices is used by this IC. These ULN2003A ICs are used to drive a varied range of loads, display drivers, line drivers etc and it also acts as relay drivers . To drive Stepper Motors we can make use of this IC. The ULN2003A pairs of darlington is rated at $500 \mathrm{~mA}$ and can resist $600 \mathrm{~mA}$ peak current. The inputs and outputs are placed reverse to each other in a layout of pin. A suppression diode is included alongwith ever diode in order to dissipate voltage spikes so as to drive inductive loads. 


\section{LabView}

LabView is a short form for Laboratory Virtual Instrument Engineering Workbench. It is obtained from national instruments. LabView is a system design development and platform which can also be made useful for image processing based visual programming language.

' $G$ ' is named as the graphical source language. It was released originally in 1986 the Apple Macintosh, LabVIEW has variety of functions and it is extremely useful for automation in the industries, controlling instruments, and acquisition of data on a wide number of platforms various versions of Linux, UNIX, Microsoft Windows, and OS X. In August 2015, the latest LabView version known as LabView 2015 was released.

Labview is manufactured in a development atmosphere for the purpose of accelerating the product usability of the scientists, programmers and software engineers. It makes it possible for the people to easily visualise, analyse, and in the creation of code by using a graphical programming syntax. It is unique and cannot be matched as it helps in reducing the test times and to gather and deliver ideas according to the data which is collected and translates these insights into reality. This software is designed to interact with any other software. Thus, it assures users to make use of all of its tools that are available be it in any platforms or alternative development.

Criticism that surrounds LabView are as follows:

\section{Dataflow Programming}

$\mathrm{G}$ is the programming language which is used in LabView. This programming language is in the form of a dataflow, the structure of a graphical block diagram that is the source code of LabView is responsible for the execution. On this source code it is possible for the programmer to connect nodes that possess different functions. This can be done by drawing wires which connects these nodes. Any node can be implemented as soon as all the data required for its execution becomes available, this is because all the wires used to interconnect nodes can generate variables. It is possible for $G$ to instinctively provide parallel execution. Thus, it becomes easier to simultaneously implement multiple inputs. To multiplex multiple threads of the operating systems over the nodes which are ready for execution. This is done by the features such as multi threading and Multi-processing and multi-threading hardware.

\section{Graphical Programming}

The front pages which is made up of user interfaces is tied to the development cycle by using LabView. The programmes or subroutines in LabView are known as virtual instruments(VIs). Each VI consists of three components: a front panel, a block diagram, and a connector panel. The connector panel is useful in representing the VI in the form of block diagrams. Controls and indicators are used in the front panel. Controls acts as inputs that is VI is supplied with the information given by the user. Indicators act as outputs that is they show results or they display, based on the inputs provided to the VI. The back panel consists of a block diagram, which includes graphical source code. The objects that are present in the front panel will be seen as terminals on the back panel. Structures and functions are located in the back panel they carry out various operations on data supply and controls to the indicators. Function palette contains the structures and functions which is easily kept on the back panel.

All together structures, functions, controls and indicators is referred to as nodes. Wires are made to interconnect the nodes. For example, three controls and an indicators can be connected through wires to perform the subtraction function so that the indicator displays the subtraction of the three controls. Thus a program or a VI is implemented with a user interface serving in the front panel or, in the back panel as a node. The connector planes determines the inputs and outputs that are placed in the front panel. A larger program can be embedded as a subroutine is implemented using visual instrument only after each VI is tested.

Thus LabView makes it possible for the non programmers to construct programs by dropping and placing representations of equipments virtually which they are already aware of. It enables to implement small purpose applications with various documentation and variety of examples. This feature obtained from its programming environment will be proved beneficial on various levels. However it can also prove to be a threat of underestimating the techniques which is required to implement a $\mathrm{G}$ programming language which is of a very high quality. The memory management and the syntax of LabView should be properly known by the programmer so as to implement algorithms which are complex and complicated or a lengthy code that is to be run on a large scale. The stand alone applications can be built by making use of the most advanced LabVIEW development systems. In addition to that, it is also enables to build applications that are distributed and theses applications communicate with each other by a client server mechanism. This is possible because the $\mathrm{G}$ programming language is parallel in nature.

\section{Benefits of using LabView}

\section{Code Compilation}

LabVIEW possesses a compiler and because of its nature and in terms of its performance it can create for the CPU platform, a native code. With the help of compilation and by interpreting its syntax the graphical source code is converted into a machine code which is executable. The syntax of the LabView is compiled into a code which is executable and inculcated during the process if editing. The source code which is executable is compiled into a single file. The $G$ language is defined can perform tasks which are common they contain code which is precompiled that runs on an executable engine on run time. The various operating systems, hardware components, graphic systems, is provided with an interface which is consistent.. The code which runs 
on various platforms in the run time environment. Compiled $\mathrm{C}$ code is faster than the LabVIEW code, but the differences in the execution speed is more than the program optimization.

\section{Large Libraries}

Numerous graphical components along with large number of functions namely analysis, statistics, data acquisition, signal generation, mathematics, signal conditioning along with numerous graphical interface elements are offered in several LabVIEW package options in its extensive libraries. Mathematics blocks for various types of functions such as filters, integration and other objects is associated generally along with capturing of data from a large number of hardware sensors.

Additionally, Math script which is implemented with various functionality is used for processing signal, mathematics and analysis using a text-based programming element given by LabView. Using script nodes which is a graphical programming language it is possible to integrate Math script, it can also use MATLAB which is compatible with the syntax.

\section{Home Bundle Edition}

Home bundle edition of a low cost LabView is provide from national instruments.

\section{Ecosystem}

It is possible for the users or provide ability to give extension for its functions which can be achieved by popularity and of the LabView software. Due to the longevity and popularity of the LabVIEW language. This results in developing of a large ecosystem comprising of the third party which is developed because of the community who has contributed. The market place has such type of ecosystem where variety of tools based on LabView is available both for free or paid.

\section{Parallel Programming}

LabVIEW is an inherently concurrent language, so it is very easy to program multiple tasks that are performed in parallel by means of multithreading. This is, for instance, easily done by drawing two or more parallel while loops. This is a great benefit for test system automation, where it is common practice to run processes like test sequencing, data recording, and hardware interfacing in parallel.

\section{Interfacing to Devices}

Interfacing to devices, cameras and instruments is supported extensively by LabVIEW.

Interfacing to hardware can be done by the users either by using specific device,high level, drivers that controls the device by providing native function codes or by simply writing down the bus commands. These bus commands include Serial, USB, GPIB.
Why is it used in this project?

The technology used is Machine vision (MV) that can be defined as the permissibility of a computer to visualise. It can be made useful in detecting, recognising and analysing the images and thus it performs image processing operations successfully. These operations are done for various type of applications such as guiding robots, controlling process, and for automatically inspecting in industry. By developing a machine vision application in Labview, we can use several image processing functions to process, analyse and display images. The camera captures images drawn on a paper and these images are processed and detected using this software. In our project, Raspberry PI acts as server and laptop acts as a client and they communicate with each other through TCP/IP. Images are captured by USB camera and they are analysed and detected using Labview which further sends this information to the server which acts as a host and sends it to the hardware devices that turns on or off accordingly.

\section{Python}

Python application is a general purpose, high level, dynamic popular that is it is widely available and used in different fields, interpreted programming language which is implemented so as to ease tasks. Code readability can be implemented using its own basic philosophy of its and it allows the programmers and users to implement its operation by expressing the ideas conceptually in fewer possible lines of code as compared to other programming languages such as $\mathrm{C}++$ or Java. For example in $\mathrm{C}$ language it requires about 4 pages to write and implement the code and the same operation can be achieved in a single page using this python application. The python programming language can successfully clear programs on both large or small scale level.

Python application supports multiple programming patterns such as, functional programming, object oriented, imperative or procedural styles. It attributes an automatic memory management and a dynamic type of system and it also includes a comprehensive large libraries.

For large number of operating systems interpreters of Python are available and thus it allows variety of systems to operate on its code. It is possible for Python code to be wrapped in a single stand alone programs that are executable for many of the operating systems and this can be done by making use of Py2exe or Pyinstaller which as third party tools. There is no need to install python interpreters as it is a self interpreted language and it is based on such software where there is no need for compilation. Thus there is no need of declaring the variables as there is no compiler involved for the interpretation of the code and thus it makes this application less time consuming to implement which results in faster processing time. 
So we configure Raspberry PI with this application as it is easier to implement and makes the processing time faster.

Python runs on top of the operating system Raspbian which is customized for Raspberry PI hardware.

It interacts with the hardware and provides information to the Python application. The libraries which we use to implement the program in our project are: time, socket, sys, signal and GPIO (General purpose I/P O/P).

\section{Working}

In this project, we use client-server architecture where client uses a network, where client uses a network as a way to connect with the server. The client uses the network to send and receive communication about its order or request. In this project PC or laptop acts as a client and Raspberry PI acts as a server, and they communicate through TCP/IP. With the help of Labview, the images seen on the camera of laptop is processed and detected and the information based on the images are sent to the server through WiFi connection. This information in the form of bytes is sent to the GPIO lines of the Raspberry PI circuit.GPIO (general purpose I/P O/P) is a generic pin integrated on the circuit,It acts as an output to the hardware.

The hardware consists of devices that are to be turned on or off. We make use of a Relay driver circuit, in which we use IC UPN 2003A that acts as an amplifier. It also consists of a voltage regulator that controls the voltage and provides $5 \mathrm{~V}$ to the IC, LED to denote the power, and relay that are connected across the terminals of the device.The output from GPIO acts as input to the IC whose output is shorted to the Relay circuit. The UPN 2003A IC amplifies from Raspberry PI to $300 \mathrm{~mA}$ which is the minimum requirement of power to run the device.We make use of Python application that runs on the Operating system of the Raspberry PI hardware. It has automatic memory management and a large comprehensive library.The program establishes the socket connection and creates a while loop so that the server is always waiting for the connection to establish between the client. So, as soon as the client sends request, the server readily accepts it and the data is transmitted from the client to the server.

As soon as an error occurs while transmission the server stops the connection and terminates the signal. Based on the input the device gets turned ON or OFF. In this project, a light bulb and motor acts as the devices which operates at $12 \mathrm{~V}$.So according to the images displayed on the camera, these devices automatically gets switched ON or OFF without making use of the five senses.

\section{TCP/IP}

\section{TCP Client/Server}

TCP SERVER

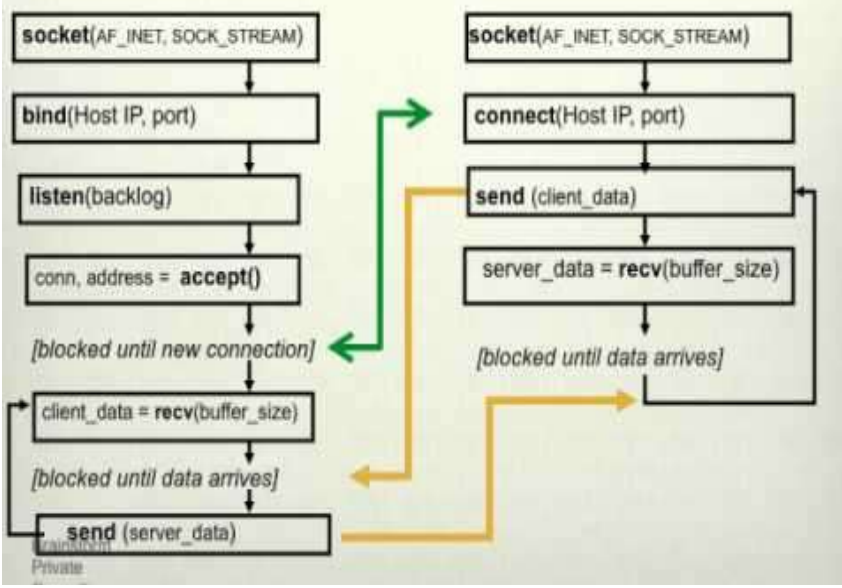

Fig-3: Flow Diagram of TCP/IP Client Server.

TCP/IP network acts as that of a telephone where we make a call by establishing the telephonic connection where someone has to start the connection by dialling the phone number and thus it works in a manner similar to that of a telephone. When the connection is established the person from other end of the conversation has to carefully listen for calls and then pick up the phone when the call the phone rings and the call comes. The IP address is different for different telephones and the port number should also vary to a particular telephonic once the call is answered. The "Server" is the computer that is listens for the call that it receives and the Client is a device that dials the phone number. Thus a server should be ready to accept the connection which it receives from the client and the client needs to know the IP address and the port number of the server where the data needs to be send and received when the connection is established. The client thus sends the call and the Server only has to listen for connections so as to either accept them or reject them.

Data can be sent in both directions once a connection through a TCP/IP port has been established between a TCP/IP server and a TCP/IP client. The network is used to send and receive the data which is the only difference. The connections is implemented and it remains as long as the client and the server are both involved. Once either the client or the server terminates the connection, the operation is stopped and the network connectivity is terminated. The most important advantage of the TCP/IP protocol is that the error checking is performed by the low level drivers that establishes the connection by exchanging data that is sending and receiving the data and thus, the interaction and also the exchange of data is guaranteed.

\section{Applications}

- It is used for the purpose of home automation( for turning on or turning of the devices)

- It can also be used in offices, schools and in corporate offices and even in the industries. 
- It features zoom.

- It is possible to get information about the product.

- People are fed with information using the newest technology.

\section{Advantages}

- $\quad$ Sixth Sense an interface which is very user friendly and its function is to integrate physical world with the digital information obtained and its objects without changing its platform.

- Sixth Sense causes computer and other devices to adapt to the needs of human rather than changing the human habits.

- It allows users to perform multi-touch and multi-user operations.

- It supports real time access.

- It is less in cost and open source type and we can map various ideas based on it.

- It can turn any environment into a display which is interactive by making use of hand gestures or other symbols and objects.

\section{Future Enhancements}

- The devices or computers can be controlled In large scale industries by using this technology.

- Mobile computing device can be altered by placing a camera and a projector inside it.

- Application of this sixth sense technology in various interests like gaming, education systems, and also for scientific reasons.

- 3D gesture tracking can be implemented using this technology.

- Allowing a disabled person to use this sixth sense to function as if its his fifth sense.

\section{CONCLUSION}

Sixth sense technology perceives the objects around us and reacts accordingly. The key in this type of approach is that the information is displayed and allows us to control or access it in the way you desire. Thus, it can be said that it has the potential of becoming the most portable and transparent user interface to access the information about the environment around us.

It makes use of the software LabView which is used for image analysing, processing and its detection and also we make use of Python application for writing the program and its implementation for the lightweight server (Raspberry Pi $\mathrm{b}+$ ). These two softwares are widely used and are developing tools. Our project deals with creating a machine vision application in order to make use of image processing functions. So it is a rather straightforward approach for the project implementation.

But it may change the approach we take to connect and communicate with the real world and provides us the complete knowledge of the physical world around us.

\section{ACKNOWLEDGEMENT}

I would like to express my sincere and deepest gratitude to Prof. Suman Wadkar for her valuable guidance, support, encouragement and immense help. Her enthusiasm and optimism made this experience both rewarding and learning.I would also like to express my sincere gratitude toProf. Rajendrakumar Khade, Head,Department of Electronics Engineering, Pillai College Of Engineering, NewPanvel for his constant support and encouragement.I express my thanks to Principal Prof. R.I.K Moorthy, Pillai College Of Engineering, New Panvelfor extending his support.I am thankful to all my friends who have helped directly or indirectly for the completion of this report.

\section{REFERENCES}

[1]. http://www.pranavmistry.com/projects/sixthsense/

[2]. www.mycollegeproject.com

[3]. http://www.engineersgarage.com/articles/sixth-sensetechnology

[4]. https://en.wikipedia.org/wiki/SixthSense

\section{BIOGRAPHIES}

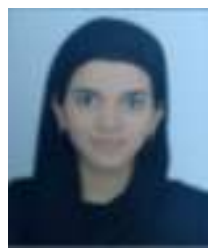

Zeba Raut, M.E student working on the project named 'Sixth sense technology for home automation' from Pillai College of Engineering. 